\title{
Estudio comparativo de la producción de polen y miel en un sistema de doble reina versus una por colmena en La Araucanía, Chile
}

\author{
Comparative study of honey and pollen production in a double queen system versus \\ one queen per hive in La Araucanía Region, Chile
}

\author{
Ramón Rebolledo R. ${ }^{1}$, Marco Riquelme C. ${ }^{1}$, Solange Huaiquil V. ${ }^{1,3}$, \\ Germán Sepúlveda Ch. ${ }^{3}$, Alfonso Aguilera P. ${ }^{2}$
}

\begin{abstract}
RESUMEN
Con el objetivo de evaluar el efecto de la cosecha del polen sobre la producción de miel, en sistemas de producción con una y dos reinas por colmena, se realizó un ensayo en la localidad de Pillanlelbún, comuna de Lautaro, Región de La Araucanía. Los tratamientos correspondieron a seis colmenas simples (una reina) con y sin trampa de polen modelo Apefiori versus seis colmenas doble reina con y sin trampa de polen. Las abejas utilizadas en el ensayo se obtuvieron a partir de núcleos formados con igual cantidad de cría y alimento, además de tener incorporadas reinas fecundadas en la temporada. Los resultados no presentaron diferencias significativas en la producción de miel para el sistema de una sola reina con y sin trampa de polen con $25 \mathrm{~kg}$ de promedio de miel en el sistema sin trampa de polen y $21 \mathrm{~kg}$ para el sistema con trampa de polen. Lo mismo ocurrió en los sistemas de doble reina con y sin trampa (51 kg sin trampa y $48 \mathrm{~kg}$ promedio con trampa de polen). Sin embargo, la producción de miel entre ambos sistemas (una y dos reinas por colmenas) presentó diferencias estadísticas significativas. Respecto a la producción de polen, esta fue estadísticamente significativa con $1,263 \mathrm{~kg}$ de polen para el sistema simple versus $2,247 \mathrm{~kg}$ en el sistema doble reina. Finalmente la cosecha del polen no afectó la producción de miel.

Palabras clave: polen, miel, trampa de polen.
\end{abstract}

\begin{abstract}
A test was done in the village of Pillanlelbún, Lautaro District, La Araucanía Region of Chile, in order to evaluate the effect on honey production of the pollen harvest, in production systems with one and two queens per hive. The treatments corresponded to six single queen hives with and without an Apefiori pollen trap versus six hives with double queen, with and without pollen trap. The bees used in the test were obtained from nuclei formed with the same quantity of breeding and food cells, as well as having fertilised queens introduced in the season. The results showed no significant differences in honey production for the system with a single queen with and without pollen trap, with an average of $25 \mathrm{~kg}$ of honey in the system without pollen traps and $21 \mathrm{~kg}$ for the system with pollen trap. The same occurred in the systems with double queens with and without pollen trap (51 kg without pollen trap and $48 \mathrm{~kg}$ average with trap). However there were significant differences in honey production between the two types of system (one and two queens per hive). The difference in pollen production was statistically significant with $1.263 \mathrm{~kg}$ of pollen in the single queen system versus $21247 \mathrm{~kg}$ in the double queen system. Finally, the pollen harvest did not affect honey production.

Key words: pollen, honey, pollen trap.
\end{abstract}

\section{Introducción}

Se define el establecimiento de una colmena plurirreina como un sistema basado en la coexistencia armoniosa de dos colonias que funcionan como una sola unidad con el fin de obtener mayor rendimiento en la producción de miel (Gutiérrez y Rebolledo, 2000; Moeller, 1987). Los sistemas plurirreina han sido ideados para obtener altos rendimientos de miel por colmena, aprovechando las grandes poblaciones de abejas que se logran consiguiendo con ello un mejor aprovechamiento del flujo de néctar (Garau, 1990).

Varios son los estudios que se refieren a la producción de miel con sistemas de doble y triple reina en los que se muestra que son más productivos

1 Universidad de La Frontera. Facultad de Ciencias Agropecuarias y Forestales. Departamento de Ciencias Agronómicas y Recursos Naturales, Temuco, Chile.

2 Universidad de La Frontera. Facultad de Ciencias Agropecuarias y Forestales. Departamento Producción Agropecuaria, Temuco, Chile.

3 Universidad de Tarapacá, Facultad de Ciencias Agronómicas, Departamento de Recursos Ambientales, Arica, Chile. 
que los sistemas tradicionales de una sola reina por colmena (Susaeta, 1968; Banker, 1975; Reid, 1979; Roma, 1981; Philippe, 1990; Villarroel et al., 1998; Gutiérrez y Rebolledo, 2000; Gris et al., 2004; Rebolledo et al., 2008).

No obstante lo anterior, los estudios se han enfocado exclusivamente a la producción de miel no tocando ningún otro aspecto de la explotación apícola tales como producción de polen y propóleos, razón por la cual el presente estudio tuvo por objetivo comparar la producción de miel y polen entre un sistema de doble reina versus un sistema tradicional de una sola reina por colmena, y evaluar el efecto de la producción de polen sobre el rendimiento de miel.

\section{Materiales y Métodos}

El ensayo se llevó a cabo en la localidad de Pillanlelbún, comuna de Lautaro, zona caracterizada por presentar una vegetación compuesta por praderas naturalizadas con especies tales como Taraxacum officinalis Weber (Diente de león); Plantago lanceolata $\mathrm{L}$. (Siete venas); Hypochoeris radicata L. (Hierba del chancho); zonas de matorrales constituidas por especies como Ulex europaeus L. (Pica pica); Rosa rubiginosa L. (Mosqueta); Rubus ulmifolius Schott (zarzamora) y por último especies arbóreas como Eucalyptus globulus Labill (Eucalipto); Prunus domestica L. (Ciruelo), Nothofagus obliqua (Mirb.) Blueme (Roble).

Para el ensayo se emplearon abejas raza Italocanadiense, pertenecientes a uno de los autores del presente trabajo.

Se usaron para el ensayo un total de 18 familias que se dispusieron de la siguiente forma: seis de ellas en colmenas convencionales tipo Langstroth que usan los apicultores en el país. Otras doce familias en cámaras de cría, especialmente construidas para el sistema de doble reina horizontal (Figura 1), respetando las medidas bases de la colmena Langstroth. Se emplearon además nueve trampas de polen modelo Apefiori disponibles en el mercado de Temuco. También se emplearon rejillas excluidoras de reinas.

Para montar el ensayo, el 20 de septiembre del 2005, se realizó una revisión del apiario y la posterior elaboración de núcleos que se usaron en el estudio. Para esto se seleccionaron las familias con mejores condiciones de cría, nivel de alimento y sanidad al momento de la inspección. La formación de los núcleos tuvo como objetivo igualar las condiciones de las familias al momento del experimento. Se elaboraron un total de 18 núcleos a los que se les adicionó una reina fecundada, los cuales fueron empleados en el ensayo.

Para lograr la fusión de las familias doble reina, previamente se construyeron seis cámaras de cría especiales (Figura 1) respetando el ancho, alto y largo de la colmena tipo Langstroth, pero que en su interior contaban con una malla metálica doble que dividía el espacio en dos partes iguales, la que permitía el paso de los olores (feromonas) con el fin de uniformar o crear un olor común en ambos lados.

El 13 de octubre de 2005 se pusieron 12 de los 18 núcleos en las cámaras especiales, tomando la precaución de no permitir el paso de las abejas entre éstas. El 17 del mismo mes se colocó en la parte superior de la cámara de cría una rejilla excluidora de reinas, permitiendo el libre tránsito de las abejas obreras, pero no el de las reinas a las respectivas alzas de miel. La necesidad de dejar un período de acostumbramiento permite evitar que las abejas y en especial las reinas se maten entre sí (Rebolledo et al., 2008).

El 5 de diciembre del 2005 se instalaron las trampas de polen modelo Apefiori en tres de las colmenas de doble reina y tres de las colmenas con una sola reina, quedando el estudio distribuido de la siguiente forma: tres colmenas doble reina sin trampa de polen y tres doble reina con trampa de polen, tres colmenas una sola reina con trampa de polen y tres de una reina sin trampa de polen, las que sirven de testigo. Las colmenas se distribuyeron completamente al azar dentro del apiario.

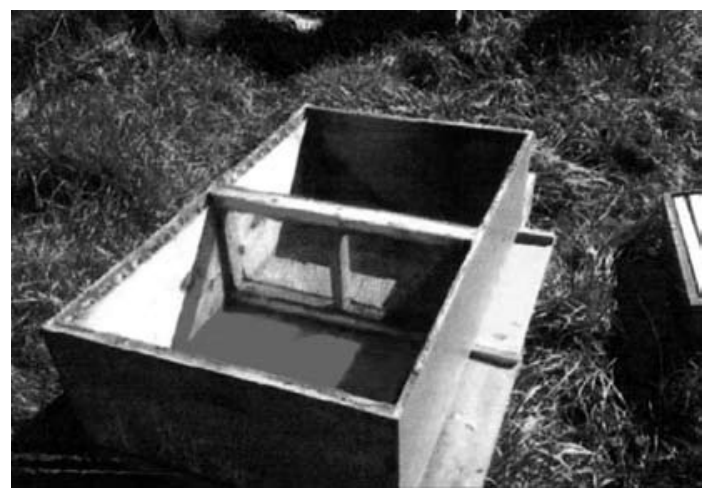

Figura 1. Cámara de cría especial para el sistema doble reina horizontal. 
Durante todo el experimento se realizó un manejo tradicional del apiario que consistió en revisiones periódicas de éste, cada 10 días aproximadamente. En las revisiones se tuvo especial cuidado de destruir todas las celdillas reales, previa revisión de la reina, además se agregaron marcos con cera estampada, así como alzas según correspondía.

El rendimiento de miel por colmena se obtuvo luego de cosechar la miel madura el día 21 de febrero de 2006. La miel cosechada fue puesta en contenedores plásticos y pesada en una balanza de una sensibilidad de $10 \mathrm{~g}$.

El polen por colmena se obtuvo luego de sumar las colectas diarias del mes de diciembre. El polen recogido fue limpiado manualmente y secado con aire caliente indirecto. Una vez procesado, se procedía a su pesaje en una balanza Soehnle con sensibilidad de un gramo.

Se utilizó un diseño factorial $(2 \times 3)$ con tres repeticiones. Los factores evaluados fueron: producción de miel y polen en a) sistema simple (una reina por colmena) y b) sistemas de doble reina.

Se realizó un análisis exploratorio de datos para verificar el cumplimiento de los supuestos de normalidad y homogeneidad de varianzas mediante la prueba de normalidad de Shapiro-Wilk y prueba de homogeneidad de varianzas de Levene. Posteriormente se realizó un análisis de la varianza y una prueba de comparación de medias de Scheffe para establecer diferencias significativas entre la producción de miel entre colmenas simples con y sin trampas de polen y colmenas doble reina con y sin trampa de polen. Finalmente se realizó una prueba t-student para muestras independientes con la finalidad de establecer diferencias de medias en el rendimiento de polen entre las colmenas simple y doble reina.

\section{Resultados y Discusión}

\section{Producción de miel en sistemas simples con y sin trampa de polen}

La producción de miel y polen en los sistemas simples (una reina por colmena), tanto con y sin trampa de polen (Figura 2), no presentó diferencias significativas entre ellos, siendo de $21 \mathrm{~kg}$ para el sistema de una reina con trampa de polen y $25 \mathrm{~kg}$ para el sistema sin trampa de polen. Sin embargo, es muy probable que estos rendimientos hubiesen sido mayores si en lugar de núcleos se hubiesen empleado familias de abejas, que salen del invierno con un buen nivel poblacional. Debido a que no se registraron diferencias significativas, se puede inferir que la extracción de polen no tuvo efecto negativo sobre la producción de miel, tal como lo señalan Cobo (1989); Crane (1990) y Shuel (1992), quienes indican que no es conveniente realizar una extracción fuerte del polen que acarrean las abejas a las colmenas, debido a un detrimento del crecimiento y a una merma en la producción de miel. Sin embargo, los resultados de la presente investigación coinciden con Del Risco (2006), quien señala que una extracción de polen, siguiendo un

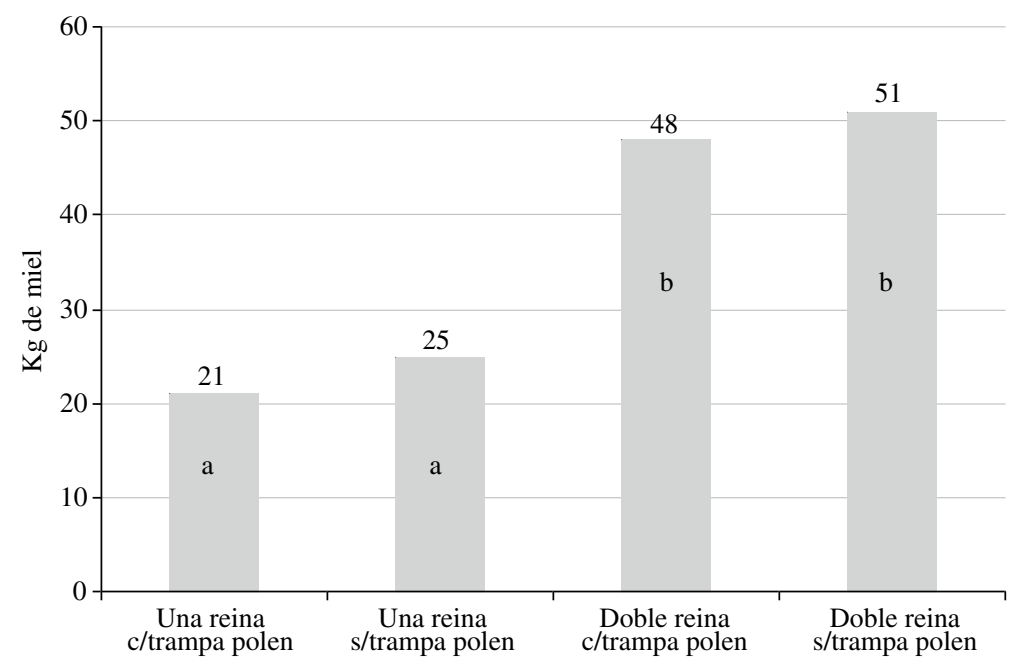

Figura 2. Promedio producción de miel en los sistemas de una y dos reinas por colmena. Temporada 2005-2006. 
Tabla 1. Producción por colmena de miel por sistema de una y doble reina con y sin trampa de polen.

\begin{tabular}{lcccc}
\hline \multicolumn{5}{c}{ Sistemas de producción de miel } \\
\hline \multirow{3}{*}{ Unidad } & $\begin{array}{c}\text { Una reina } \\
\text { sin trampa con trampa } \\
\text { de polen }\end{array}$ & $\begin{array}{c}\text { Una polen } \\
\text { sin trampa }\end{array}$ & $\begin{array}{c}\text { con pole reina } \\
\text { de polen }\end{array}$ & de polen \\
\hline Colmena 1 & $25 \mathrm{~kg}$. & $21 \mathrm{~kg}$. & $55 \mathrm{~kg}$. & $54 \mathrm{~kg}$. \\
Colmena 2 & $22 \mathrm{~kg}$. & $23 \mathrm{~kg}$. & $52 \mathrm{~kg}$. & $48 \mathrm{~kg}$. \\
Colmena 3 & $25 \mathrm{~kg}$. & $19 \mathrm{~kg}$. & $46 \mathrm{~kg}$. & $41 \mathrm{~kg}$. \\
\hline
\end{tabular}

manejo adecuado, no perjudica el normal desarrollo de la colmena. En efecto, durante la evaluación no se apreciaron grandes diferencias en la cantidad de crías, abejas, reservas de alimento y operculado de la miel.

\section{Producción de miel en los sistemas de doble reina con y sin trampa de polen}

Respecto a la producción de miel y polen en los sistemas de doble reina con y sin trampa de polen (Figura 2), ocurrió una situación similar a la descrita anteriormente. El promedio de producción de miel en las colmenas doble reina con trampa de polen fue de $48 \mathrm{~kg}$ y de $51 \mathrm{~kg} /$ colmena en las colmenas sin trampa de polen, no presentado diferencias significativas entre ambos sistemas, tampoco en la cantidad de cría, número de abejas, reservas alimenticias y operculado de la miel.

Estos resultados concuerdan con lo reportado por Villarroel (1991); Gutiérrez \& Rebolledo (2000) y Gris et al. (2004), quienes determinaron que los sistemas de doble reina producen, significativamente, mayor cantidad de miel por temporada y se explica por el mayor número de abejas presentes en el sistema de doble reina, que según Philippe (1990), Gris et al. (2004) y Pesante (2006), es uno de los factores que más influye en la producción de miel.

La ausencia de diferencias significativas de rendimiento de miel entre colmenas doble reina con y sin trampa de polen confirma que la extracción usando trampas modelo Apefiori no produjo efecto sobre las colonias. Al respecto, Moreno (2001), Salamanca et al. (2004) y Castillo (2006) señalan que las trampas pueden producir daño en alas de las abejas y, si bien es cierto que las trampas disminuyen la entrada de polen a la colmena, la colonia se encarga de equilibrar esta situación, disponiendo de más abejas pecoreadoras en faenas de recolectar polen hasta completar los 30 a 50 requeridos por colmena y que, a diferencia del néctar, el polen no es un producto escaso en la naturaleza y es posible contar con él todo el año con variaciones según la raza de abejas (Bazurro, 1999).

En relación a los rendimientos alcanzados por las colmenas simples, estos fueron mayores a los obtenidos por Villarroel et al. (1998) y Gutiérrez y Rebolledo (2000), quienes obtuvieron un promedio de 14 y $8 \mathrm{~kg}$, respectivamente, en los estudios realizados en la región de La Araucanía. Lo mismo ocurrió para los sistemas doble reina evaluados por estos mismos autores, quienes lograron rendimientos promedio de 36 y $45 \mathrm{~kg}$ respectivamente, mientras que en el presente estudio el promedio fue de $49 \mathrm{~kg}$. Esta diferencia se explica en el efecto que tuvo la utilización de reinas de la temporada que tienen como características mayores tasas de postura de huevos (Bazurro, 1999 y Pesante, 2006), pudiendo poner según Guzmán (2006) hasta un 39\% más; el mismo autor señala que el hecho de tener reinas nuevas puede incrementar la producción de miel entre 15 a $30 \%$.

\section{Producción de polen en sistema simple versus sistema de doble reina horizontal}

La producción promedio de polen (Figura 3, Tabla 2) presentó diferencias estadísticas significativas, siendo mayor en las colmenas con doble reina. La mayor producción que obtuvo el sistema doble reina tiene la misma explicación que en caso de la miel; es decir, la superior cantidad de abejas pecoreadoras que se alcanza con este sistema permite una mayor entrada de polen a la misma.

En relación al volumen de polen cosechado, debe destacarse que las cifras corresponden a un mes de colecta, diciembre 2005, época en que la

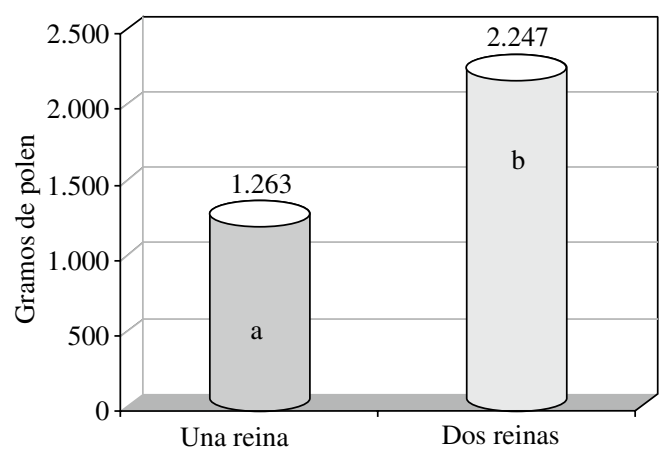

Figura 3. Producción promedio de polen en los sistemas de una y dos reinas por colmena. Diciembre 2005 a enero 2006. 
Tabla 2. Producción por colmena de polen en ambos sistemas de una y dos reinas por colmena.

\begin{tabular}{lcc}
\hline & \multicolumn{3}{c}{ Sistemas de producción de polen } \\
\hline Unidad & Una reina & Dos reinas \\
\hline Colmena 1 & 1.203 gr. & 2.500 gr. \\
Colmena 2 & $1.455 \mathrm{gr}$. & 2.016 gr. \\
Colmena 3 & 1.130 gr. & 2.225 gr. \\
\hline
\end{tabular}

floración del sector comenzó a declinar producto de la sequía, que afectó la floración de importancia apícola bajando fuertemente y con ello el término anticipado la temporada productiva.

\section{Conclusiones}

La producción de polen, durante el periodo de evaluación, en un sistema simple comparado con un sistema de doble reina no tuvo incidencia en el rendimiento de miel.

El sistema de doble reina permitió obtener producciones de polen y miel significativamente superiores al sistema de una reina por colmena.

La cosecha de polen no afectó la producción de miel y se recomienda su uso en sistemas de doble reina horizontal, que presentan una mayor producción de miel y polen.

\section{Literatura Citada}

Banker, R

1975 Manejo de colonias con dos reinas. En Dadant e Hijos, La colmena y la abeja melífera. Hemisferio Sur. Montevideo. Uruguay, pp. 522-531.

Bazurro, J.

1999 La importancia de la alimentación en el manejo productivo de las colmenas. División promoción a la producción. Departamento de Apicultura. Canelones. Uruguay. 33 p.

Castillo, R.

2006 Producción de polen en América Latina. Disponible en http://www.noticiasapicolas.com/anterirores9.htm. Visitada 10 de marzo 2006.

Cobo, A.

1989 El polen, recolección y manejo. En, M. Flores, Curso de apicultura. Edición Técnica. Ministerio de Agricultura, Pesca y Alimentación. Madrid, España, pp. 221-227.

Crane, E.

1990 Bees and beekeeping. Science, Practice and World Resources. Comstock Publishing Associates. New York. 614 p.

Del Risco

2006 Polen-Pan de abejas: Composición, Nutrición, Acción en la Salud Humana y Microbiología. Disponible en http:// www.culturaapicola.com.ar/apuntes/alimentacion/35_polen_ pan_de_abejas.odc. Visitado 20 de abril 2008.

Garau, J.

1990 Curso superior de apicultura. Palma de Mallorca. Baleares España. 603 p.

Gris, A.; Guzmán, E.; Correa, A. \& Zoraya, A.

2004 Efecto del uso de dos reinas en la población, peso, producción de miel y rentabilidad de colonias de abejas (Apis mellifera) en el altpiplano de México. Disponible en http://www. Tecnicapecuaria.org.mx/publicaciones/ publicacion04.php?IDPublicacion=437. Consultado $23 \mathrm{de}$ noviembre 2004.

Gutiérrez, J. \& Rebolledo, R.

2000 Comparación de la producción de miel en dos sistemas de doble reina y un sistema tradicional de una reina por colmena. Agro Sur 28 (2): 10-14.
Guzmán, E.

2006 Manual para la cría de abejas reina. Programa Nacional de Apicultura. SAGARPA Disponible en http://www.Info-bee.com. ar/files/docs/cria_reinas.pdf. Visitado 12 de marzo 2008.

Moeller, M.

1987 Administración de colmenas para lograr un alto rendimiento de miel. En, Mc GREGOR La apicultura en los Estados Unidos. Limusa, México, pp. 23-30.

Moreno, J.

2001 Manual de enfermedades de las abejas. Publicación del Servicio Agrícola y Ganadero. Chile. 49 p.

Pesante, D.

2006 Factores primarios que afectan la cantidad de miel almacenada por la colonia de abejas en un ambiente subtropical. Disponible en http://www.academic.uprm.edu/dpesante) docs-apicultura/factores-primarios-porudcion. PDF.

Philippe, J.

1990 Guía del apicultor. Ediciones Mundi Prensa. Madrid, España. 376 p.

Rebolledo, R.; Guiñez, C.G.; Araneda, X. \& Aguilera, A. 2008 Estudio comparativo de la producción de miel con una y tres reinas por colmena en la zona de Nueva Imperial, Chile. Idesia 26 (2): 19-25.

Reid, M.

1979 Requeening honeybee colonies without dequeening using protected queen cells. New Zealand Beekeper. 40 (3): $15-17$

Roma, A.

1981 Explotación intensiva del colmenar. Sintes. Barcelona. $355 \mathrm{p}$.

Salamanca, G.; Hernández \& Vargas, E.

2004 El polen en el sistema de puntos críticos, cosecha, propiedades y condiciones de manejo. Disponible en: http:// beekeeping.com/articulos/salamanca/polen_apicultura.htm. Visitado 12 de septiembre 2008.

Susaeta, M.

1968 Aplicación de un sistema de dos reinas para la polinización de alfalfa y trébol rosado. Investigación y Progreso Agrícola. Instituto de Investigaciones Agropecurias. Santiago, Chile. 2: $9-12$. 
Shuel, R.

1992 The production of nectar and pollen. In Dadant \& Sons The hive and the honey bee. M \& W Graphics. Michigan USA, pp. 401-425.
Villarroel, D.; Rebolledo, R. \& Aguilera, A

1998 Estudio comparativo de producción de miel con una y dos reinas por colmena en la zona de Nueva Imperial, IX Región, Chile. Agro Sur 26 (2): 121-126. 